\title{
Association of a locus on rat chromosome 4 with anxiety-related behaviors in two selectively bred rat lines
}

\author{
Thaïs M. Hameister ${ }^{1}$, Geison S. Izídio ${ }^{1}$, Victor H. Valiati ${ }^{2}$ and André Ramos ${ }^{1}$ \\ ${ }^{1}$ Laboratório de Genética do Comportamento, Departamento de Biologia Celular, Embriologia e Genética, \\ Universidade Federal de Santa Catarina, Florianópolis, SC, Brazil. \\ ${ }^{2}$ Laboratório de Biologia Molecular, Universidade do Vale do Rio dos Sinos, São Leopoldo, RS, Brazil.
}

\begin{abstract}
The Floripa $\mathrm{H}$ and $\mathrm{L}$ rat lines, selected for high and low locomotion in the central aversive area of an open field, a widely used emotionality test, were proposed as a model for studying the genetic basis of anxiety. The present study aimed to verify if the QTL Ofil1, mapped to rat chromosome 4 and previously identified as being related to emotionality in another population of rats, contributes to the behavioral variability observed in the Floripa rat lines. To this purpose, rats of five generations of selective breeding were genotyped for two polymorphic markers, D4RAT59 and D4MGH27, flanking Ofil1. Changes in genotype and allele frequencies throughout generations were evaluated in both $\mathrm{H}$ and $\mathrm{L}$ lines, in order to assess if the bidirectional selection based on behavioral scores induced divergent changes in the genotype of this genome region. There were significant changes in genotype frequencies for both molecular markers, however, only the genotype variations of the D4RAT59 marker were significantly correlated with the variations in the selected phenotype. This result suggests that the region of the genome near D4RAT59 contains one or more genes contributing to the interindividual variation in central locomotion in the open field test.
\end{abstract}

Key words: QTL, emotionality, rat lines, behavior genetics, open field.

Received: February 15, 2008; Accepted: July 8, 2008.

\section{Introduction}

Anxiety is a common psychiatric disorder that affects nearly one third of the human population sometime in life (Anagnostaras et al., 1999; Finn et al., 2003). Anxietyrelated disorders and behaviors are highly complex traits that are still poorly understood, as they result from the interaction of multiple unknown genes with countless environmental factors (Finn et al., 2003). Animal models can provide valuable information about the underlying genetic, neurobiological and molecular factors of complex psychological traits such as anxiety. For example, Quantitative Trait Locus (QTL) analysis using laboratory animals enables the location of the main genome regions which contribute to interindividual variability in anxiety-related behaviors. Once identified, these QTLs become important starting points to home in on candidate genes for different psychopathologies (Finn et al., 2003; Willis-Owen and Flint, 2007).

Send correspondence to André Ramos. Laboratório de Genética do Comportamento, Departamento de Biologia Celular, Embriologia e Genética, Centro de Ciências Biológicas, Universidade Federal de Santa Catarina, 88040-900 Florianópolis, SC, Brazil. E-mail: andre@ccb.ufsc.br.
Several QTLs for anxiety-related traits have been identified in rodents during the past decade (Flint, 2003). In 1999, Ramos and colleagues identified, for the first time, genome regions influencing anxiety-related behaviors in rats (Ramos et al., 1999). That study, based on the F2 generation of two inbred rat strains, Lewis (LEW) and Spontaneously Hypertensive Rats (SHR), allowed the mapping of a locus on chromosome 4 that exerted a major influence on the animals' locomotion in the central aversive area of an open field (OF) test. The influence of this locus, named Ofil1 (open field inner locomotion 1), was later confirmed, not only for the levels of central OF locomotion, but also for alcohol preference, using recombinant lines derived from LEW and SHR rats (Mormède et al., 2002; Vendruscolo et al., 2006). Up to now, the genes involved and the relevance of this locus for other populations of laboratory rats remain unknown. However, other QTLs near this region have been shown to affect stress responses and alcohol drinking in different strains of rats (Bice et al., 1998; Carr et al., 1998; Terenina-Rigaldie et al., 2003; Potenza et al., 2004).

Given the potential importance of the locus Ofill for the modulation of behavioral processes, including anxiety-related ones, it is essential to learn more about it, both in 
terms of its map position and of its relevance for other populations and/or species of animals. Therefore, the present study aimed to determine whether the QTL Ofil1 would also influence anxiety-related behaviors in a different population of rats, represented here by the Floripa $\mathrm{H}$ and $\mathrm{L}$ rat lines. These lines were selectively bred, respectively, for high and low levels of locomotion in the central area of the $\mathrm{OF}$, an experimental measure of fearfulness in rodents (Ramos and Mormède, 1998). After five generations, the $\mathrm{H}$ and L lines differed from each other not only for the selected behavior but also for other experimental indices of anxiety, such as the approach towards the open arms of an elevated plus maze and the white compartment of a black/white box (Ramos et al., 2003). In all cases, Floripa L rats showed higher avoidance of these aversive areas (a sign of higher anxious-like behavior) than their H-line counterparts.

In order to determine whether the bidirectional selection based on the animals' behavioral phenotype induced parallel changes in the genotype around the locus Ofill, animals of both $\mathrm{H}$ and $\mathrm{L}$ lines from the first five generations of selective breeding ( $\mathrm{S} 0$ to $\mathrm{S} 4$ ) were genotyped in relation to two polymorphic markers flanking the peak of the previously described QTL. Changes in genotype and allele frequencies throughout the process of selection were evaluated in both rat lines.

\section{Material and Methods}

\section{Subjects}

The DNA samples used in the present study had been collected and stored at $-20{ }^{\circ} \mathrm{C}$ during the years 2000 and 2001. At that time, animals from the first five generations (S0 to S4) of the Floripa $\mathrm{H}$ and $\mathrm{L}$ rat lines were raised in our laboratory, being phenotyped at 8-9 weeks of age in several behavioral tests of anxiety/emotionality and then sacrificed, having their liver and spleen removed for further DNA extraction. The selective breeding procedure was described elsewhere (Ramos et al., 2003). Briefly, a genetically heterogeneous stock of rats (named S0) was obtained through the intercross of three commercial lines: Wistar, Hooded and Lewis. Male Wistar rats were mated with female Hooded rats and their female offspring were mated with male Lewis rats. Albino males and females of this last generation were intercrossed to produce a heterogeneous S0. Males and females (30/sex) of this generation were tested in an OF test, and the 10 animals (5/sex) with the highest and the 10 animals (5/sex) with the lowest scores for central locomotion were mated to establish the new $\mathrm{H}$ and L lines, respectively. At each successive generation, the two lines developed independently, with 5 males and 5 females with extreme scores for OF central locomotion being chosen from each line as breeders for the following generation. Brother-sister mating was avoided.

\section{Genotyping}

Genomic DNA was extracted from spleen and/or liver tissues using a DNAzol commercial kit (Invitrogen Life Technologies, São Paulo, Brazil). Primers for the microsatellite markers D4MGH27 and D4RAT59, mapped to chromosome 4 near the QTL Ofill (Ramos et al., 1999), were purchased from WMED-World Medical Ltda (São Paulo, Brazil). The primer sequences for D4RAT59 were: (F) 5'-GCG GAA TGA TAG TTA CTA CGG C-3' and (R) 5'-GCA GTG TGT TTG GGG TAG CT-3'; and for D4MGH27: (F) 5' - TCC TTC ACA TAC ATG TGC ATA CC-3' and (R) 5'- TGA GAA GGG CTG TCA GTG G-3'.

Genotypes were determined for all animals of each generation, regardless of their phenotype, except for generation S3. In this particular case, DNA samples from unselected animals were not available and thus only the 10 rats from the $\mathrm{H}$ line with the highest scores and the 10 rats from the L line with the lowest scores for central OF locomotion were genotyped. Genotyping was performed by polymerase chain reaction (PCR), followed by electrophoresis either in $3 \%$ agarose or $8 \%$ polyacrylamide gels. In a $20 \mu \mathrm{L}$ reaction volume, $5 \mu \mathrm{L}$ of genomic DNA, $5 \mu \mathrm{L}$ of markerspecific oligonucleotides and $10 \mu \mathrm{L}$ of PCR $2 \mathrm{x}$ solution were mixed. To the previously prepared PCR $2 \mathrm{x}$ buffer, $0.076 \mu \mathrm{L}$ of Taq polymerase (Promega) was added per reaction. The PCR program was: (i) one cycle at $96{ }^{\circ} \mathrm{C}$ for $5 \mathrm{~min}$; (ii) 35 cycles at $92{ }^{\circ} \mathrm{C}$ for $30 \mathrm{~s}$; 55.8 or $61.5^{\circ} \mathrm{C}$ (for markers $\mathrm{D} 4 \mathrm{MGH} 27$ and D4RAT59, respectively) for $1 \mathrm{~min}, 72^{\circ} \mathrm{C}$ for $30 \mathrm{~s}$; and (iii) one cycle at $72^{\circ} \mathrm{C}$ for $2 \mathrm{~min}$. Alleles present in the population were first identified in the S0 generation, using $8 \%$ polyacrylamide gels. DNA samples from inbred LEW and SHR rats, in which the allele sizes were known for both markers (Rat Genome Database), were used as references. Thus, the sizes of the alleles found in the Floripa rat lines were estimated using the known sizes of the reference LEW and SHR PCR products. After that, all DNA samples from generations S0 to S4 were genotyped, either in 3\% agarose gels stained with ethidium bromide (for D4MGH27) or in $8 \%$ polyacrylamide gels stained with silver (for D4RAT59).

\section{Statistical analyses}

Based on the number of animals obtained for each of the possible genotypes at D4RAT59 and D4MGH27, the allele frequencies were determined and the expected numbers of individuals per genotype class were calculated according to the hypothesis of Hardy-Weinberg equilibrium (Falconer, 1987). The expected values for generations S1 to S4 were estimated using the allele frequencies of the previous generation, as described by Kondaurova et al. (2006). These data were tested by chi-square analysis, separately for each rat line and each generation, using the Yates correction in view of the small sample sizes. Values were considered significant when $\mathrm{p}<0.05$. In order to assess 
progressive changes in the genotype proportions throughout the selection process, contingency chi-squares were also calculated for the markers D4RAT59 ( df = 20) and $\mathrm{D} 4 \mathrm{MGH} 27$ ( $\mathrm{df}=8$ ), separately for each rat line, considering the number of animals per genotype across five generations (S0-S4).

Correlation analyses were performed to assess genotype-phenotype correlations throughout generations. For the phenotype, we used the previously known mean values of central locomotion in the OF obtained from generations S0 to S4 (Ramos et al., 2003). For the genotype, we considered the frequencies of $\mathrm{D} 4 \mathrm{MGH} 27$ allele 3 and of D4RAT59 allele 1, in each generation. In order to minimize the fluctuating, non-genetic effects of seasonal and experimental factors on the behavior of the different selected generations, we performed a Pearson correlation analysis between phenotype and genotype, using the delta values between lines. To this end, we considered the differences between lines $(\mathrm{H}-\mathrm{L})$ for central OF locomotion and for allelic frequencies across generations. For all analyses, the software Statistica 7.0 was used.

\section{Results}

Among the DNA samples of the S0 population, which gave origin to both Floripa $\mathrm{H}$ and $\mathrm{L}$ rat lines, three different alleles were identified for the marker D4RAT59 and two were found for the marker D4MGH27 (see Table 1). For each of these microsatellites, the alleles were numbered in an increasing order, from 1 to $\mathrm{n}$, according to their sizes (these numbers included the alleles from the reference strains LEW and SHR). As shown in Table 1, for D4RAT59, the S0 population contained the alleles 1, 3 and 4, and for D4MGH27, it contained the alleles 2 and 3. For D4Rat59, allele 4 was 2-4 nucleotides longer than allele 3, which was longer than the SHR reference allele (174 bp).
Table 1 - Alleles identified for each of the two microsatellite markers in the S0 population, which gave origin to the Floripa $\mathrm{H}$ and $\mathrm{L}$ rat lines, and in the reference inbred strains LEW e SHR.

\begin{tabular}{lcccc}
\hline Marker & Allele 1 & Allele 2 & Allele 3 & Allele 4 \\
\hline D4Rat59 & $\mathbf{1 6 8}$ bp & 174 bp & $+\mathbf{1 7 4}$ bp & $+\mathbf{1 7 4}$ bp \\
& (LEW e S0) & (SHR) & (S0) & (S0) \\
D4Mgh27 & 252 bp & $+\mathbf{2 5 2}$ bp & $\mathbf{2 5 8}$ bp & \\
& (SHR) & (S0) & (LEW e S0) & \\
\hline
\end{tabular}

Alleles from 1 to 4 are ordered by size. The populations where each of the alleles was identified are shown in parenthesis. The alleles found in the population S0 are indicated in bold. + means "larger than".

The genotype frequencies and the chi-square test results for the Hardy-Weinberg equilibrium in the Floripa $\mathrm{H}$ and $\mathrm{L}$ rat lines, throughout the first generations of selective breeding, are summarized in Tables 2 and 3, respectively, and the corresponding allele frequencies are shown in Figure 1. Except for S3, where only the selected animals were genotyped, in all other generations all rats were genotyped, regardless of their behavioral phenotype.

Figure 1 suggests consistent changes in the allele frequencies of both markers throughout generations of selection, with the frequency of some alleles increasing in one line and decreasing in the other line. In addition, chi-square analyses allowed rejection of the equilibrium hypothesis in most generations of $\mathrm{H}$ and $\mathrm{L}$ rats (i.e., $\mathrm{S} 1, \mathrm{~S} 3$ and $\mathrm{S} 4$ of the $\mathrm{H}$ line and S3 and S4 of the L line for D4RAT59; S1, S2 and $\mathrm{S} 3$ of the $\mathrm{H}$ line and S2 and S4 of the L line for D4MGH27) (Tables 2 and 3). Moreover, the contingency chi-square tests performed within lines but across generations were shown to be significant in both lines and for both markers, demonstrating very clearly that the genotype frequencies did vary significantly between generations in both rat lines. The chi-square values were as follows. $\mathrm{H}$ line: $(\mathrm{X} 2=37.88$, $\mathrm{df}=20, \mathrm{p}<0.01$ for D4RAT59; and X2 $=34.91$, $\mathrm{df}=8$, $\mathrm{p}<0.001$ for D4MGH27) and L line: $(\mathrm{X} 2=83.00, \mathrm{df}=20$,

Table 2 - Total number of individuals per genotype class and chi-square for Hardy-Weinberg equilibrium, for each generation of Floripa H rats, in relation to two markers for the QTL Ofill.

\begin{tabular}{|c|c|c|c|c|c|c|c|c|c|c|c|c|}
\hline \multirow[b]{2}{*}{ Generation } & & \multicolumn{7}{|c|}{ D4RAT59 genotype } & \multicolumn{4}{|c|}{ D4MGH27 genotype } \\
\hline & & $1 / 1$ & $1 / 3$ & $1 / 4$ & $3 / 3$ & $3 / 4$ & $4 / 4$ & $\mathrm{X} 2(\mathrm{df}=4)$ & $2 / 2$ & $2 / 3$ & $3 / 3$ & $\mathrm{X} 2(\mathrm{df}=1)$ \\
\hline S0 & Observed & 16 & 25 & 7 & 2 & 0 & 2 & & 8 & 27 & 17 & \\
\hline \multicolumn{13}{|l|}{$\mathrm{N}=52$} \\
\hline S1 & Observed & 6 & 8 & 9 & 2 & 0 & 0 & $16.27^{*}$ & 3 & 7 & 15 & $7.15^{*}$ \\
\hline $\mathrm{N}=25$ & Expected & 9.61 & 8.68 & 3.10 & 1.96 & 1.40 & 0.25 & & 4.20 & 12.10 & 8.70 & \\
\hline S2 & Observed & 9 & 4 & 3 & 0 & 0 & 0 & 8.97 & 0 & 2 & 14 & $8.41 *$ \\
\hline $\mathrm{N}=16$ & Expected & 5.38 & 4.45 & 3.34 & 0.92 & 1.38 & 0.53 & & 1.08 & 6.16 & 8.76 & \\
\hline S3 & Observed & 6 & 3 & 1 & 0 & 0 & 0 & $9.93 *$ & 0 & 2 & 8 & $7.62 *$ \\
\hline $\mathrm{N}=10$ & Expected & 6.08 & 2.03 & 1.41 & 0.17 & 0.23 & 0.08 & & 0.04 & 1.12 & 8.84 & \\
\hline S4 & Observed & 23 & 3 & 1 & 0 & 0 & 0 & $14.57^{*}$ & 0 & 3 & 24 & 3.46 \\
\hline$N=27$ & Expected & 17.28 & 6.48 & 2.16 & 0.61 & 0.41 & 0.06 & & 0.27 & 4.86 & 21.87 & \\
\hline
\end{tabular}

*significant at $\mathrm{p}<0.05$. 
Table 3 - Total number of individuals per genotype class and chi-square for Hardy-Weinberg equilibrium, for each generation of Floripa L rats, in relation to two markers for the QTL Ofill.

\begin{tabular}{|c|c|c|c|c|c|c|c|c|c|c|c|c|}
\hline \multirow[b]{2}{*}{ Generation } & & \multicolumn{7}{|c|}{ D4RAT59 genotype } & \multicolumn{4}{|c|}{ D4MGH27 genotype } \\
\hline & & $1 / 1$ & $1 / 3$ & $1 / 4$ & $3 / 3$ & $3 / 4$ & $4 / 4$ & $\mathrm{X} 2(\mathrm{df}=4)$ & $2 / 2$ & $2 / 3$ & $3 / 3$ & $\mathrm{X} 2(\mathrm{df}=1)$ \\
\hline S0 & Observed & 16 & 25 & 7 & 2 & 0 & 2 & & 8 & 27 & 17 & \\
\hline \multicolumn{13}{|l|}{$\mathrm{N}=52$} \\
\hline S1 & Observed & 10 & 12 & 5 & 2 & 5 & 0 & 7.16 & 6 & 19 & 9 & 1.21 \\
\hline $\mathrm{N}=34$ & Expected & 13.07 & 11.80 & 4.22 & 2.67 & 1.90 & 0.34 & & 5.72 & 16.45 & 11.83 & \\
\hline S2 & Observed & 11 & 8 & 2 & 4 & 0 & 1 & 6.96 & 10 & 9 & 7 & $4.57 *$ \\
\hline $\mathrm{N}=26$ & Expected & 7.58 & 8.70 & 4.21 & 2.50 & 2.42 & 0.59 & & 5.50 & 12.92 & 7.58 & \\
\hline S3 & Observed & 1 & 5 & 4 & 0 & 0 & 0 & $20.43^{*}$ & 5 & 3 & 2 & 1.80 \\
\hline $\mathrm{N}=10$ & Expected & 3.84 & 3.84 & 0.87 & 0.96 & 0.43 & 0.06 & & 3.13 & 4.93 & 1.94 & \\
\hline S4 & Observed & 5 & 5 & 2 & 6 & 0 & 5 & $32.68 *$ & 6 & 16 & 1 & $6.17 *$ \\
\hline $\mathrm{N}=23$ & Expected & 6.96 & 6.32 & 5.06 & 1.44 & 2.30 & 0.92 & & 9.72 & 10.46 & 2.82 & \\
\hline
\end{tabular}

*significant at $\mathrm{p}<0.05$.

$\mathrm{H}$ line (D4RAT59)

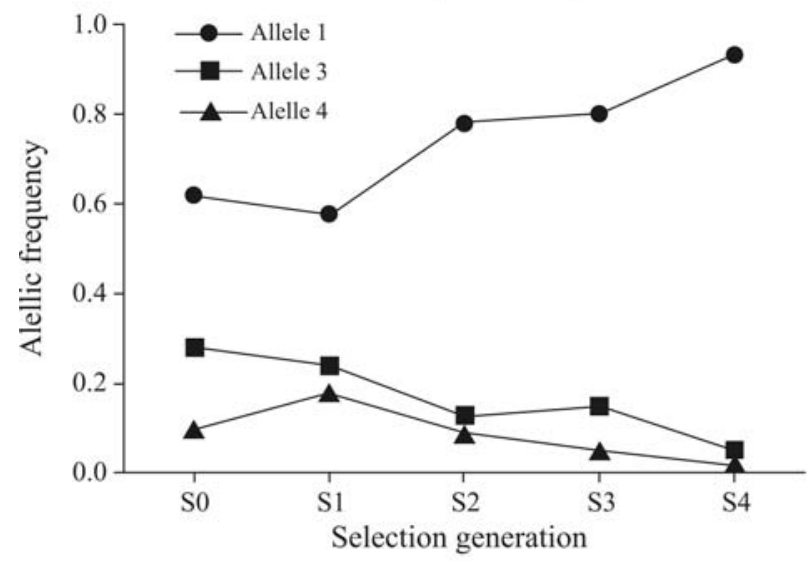

L line (D4RAT59)

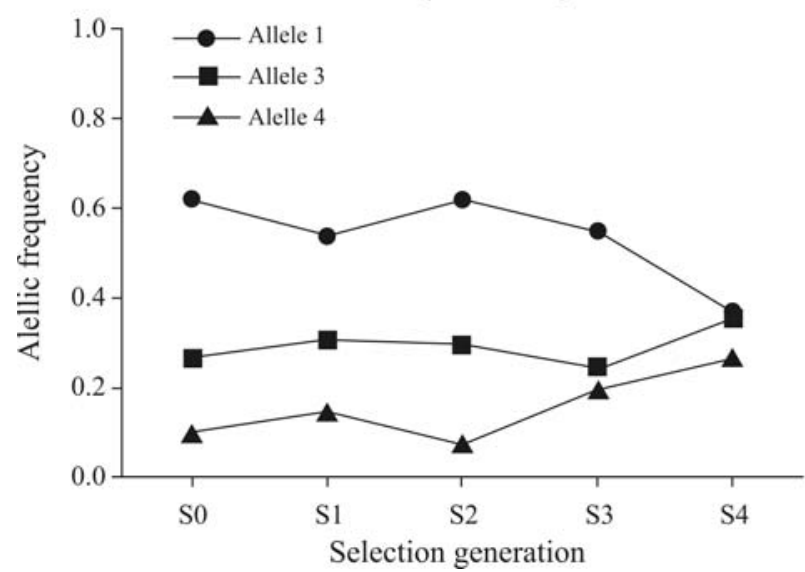

$\mathrm{H}$ line (D4MGH27)

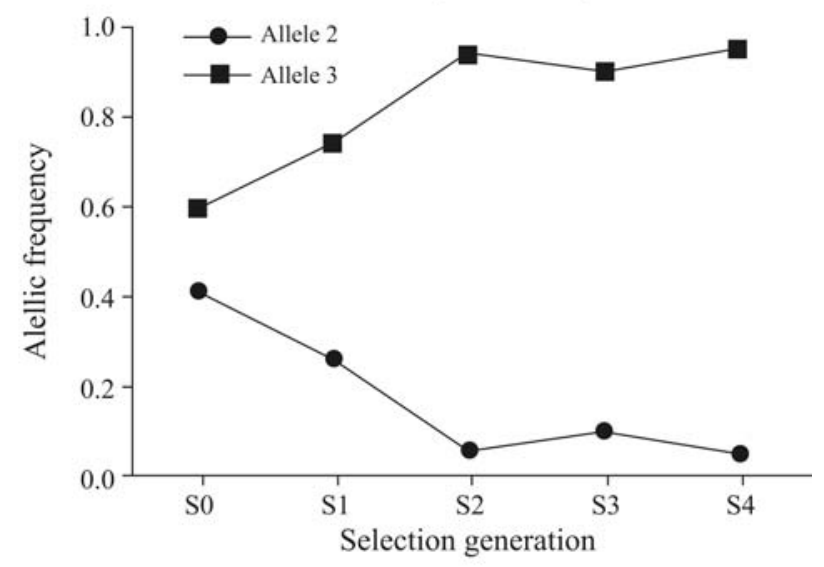

L line (D4MGH27)

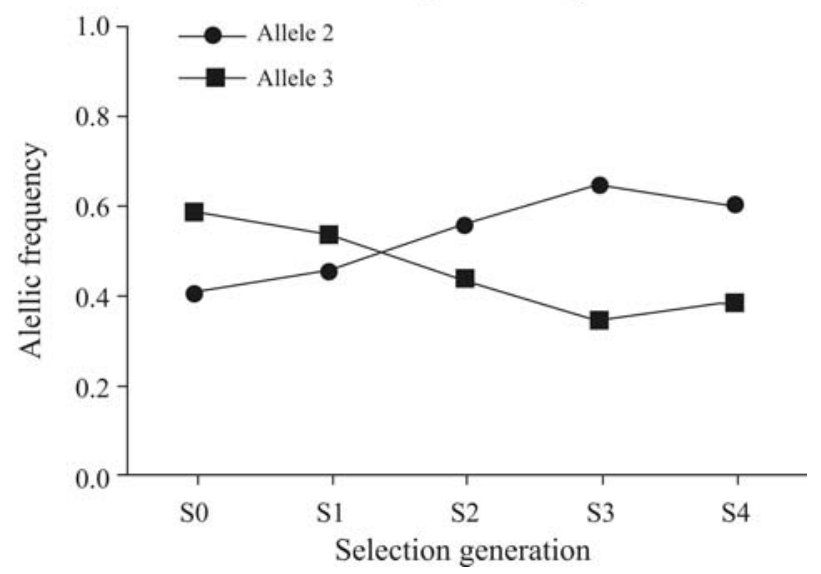

Figure 1 - Frequencies of alleles 1, 3 and 4 of the microsatellite marker D4RAT59 (left panels), and of alleles 2 and 3 of the microsatellite marker D4MGH27 (right panels), both located on rat chromosome 4, from generation S0 to S4 of selective breeding in the Floripa H (top) and L (bottom) rat lines. 
$\mathrm{p}<0.01$ for D4RAT59; and $\mathrm{X} 2=19.96, \mathrm{df}=8, \mathrm{p}<0.05$ for D4MGH27).

Figure 2 illustrates the comparative frequencies of allele 1 at D4RAT59 and allele 3 at D4MGH27 in both the $\mathrm{H}$ and $\mathrm{L}$ rat lines simultaneously. This comparison shows that the changes in allele frequencies were consistent with the hypothesis that the genome region near these markers is associated with the behavioral trait under selection. In spite of evolving independently, the two populations showed changes in opposite directions, as expected.

In order to investigate whether these changes were related to the behavioral changes of the Floripa $\mathrm{H}$ and $\mathrm{L}$ rats during selection, we performed a Pearson correlation analysis of the genotype and phenotype delta values, using the between-line differences in the frequencies of the two aforementioned alleles and the between-line differences in the $\mathrm{OF}$ behavior from generation $\mathrm{S} 0$ to $\mathrm{S} 4$. The results showed that only the frequency of allele 1 at D4RAT59 was significantly correlated with behavioral variation (Figure 2).

\section{Discussion}

The main aim of this study was to experimentally investigate the association of the QTL Ofill on chromosome 4 , previously identified as influencing emotionality in the F2 generation of the inbred rat strains LEW and SHR (Ramos et al., 1999), with emotional reactivity in a different population of rats, selectively bred for high and low scores of central locomotion in the OF test (Ramos et al., 2003). These new lines, named Floripa $\mathrm{H}$ and L, respectively, derived from a genetically heterogeneous stock (named S0) that was produced for this purpose through the intercross of two outbred rat lines (Wistar and Hooded) and one inbred strain (LEW). This initial population was expected to show a great variability in both behavioral phenotypes and genotypes throughout the entire genome. As the selected trait is polygenic, alleles of polymorphic genes (and their neighboring markers) that contribute to increase the trait were expected to show an increasing frequency across generations in the $\mathrm{H}$ line and a decreasing frequency in the L line, with the opposite being expected for those alleles causing a decrease in the trait. On the other hand, genome regions containing no relevant polymorphism in regards to the selected behavior should either remain constant or display random changes in allele frequency throughout generations.

After its first description, in 1999, the aforementioned QTL was confirmed as affecting the same behavior in two independent studies (Mormède et al., 2002; Vendruscolo et al., 2006). Both of them, however, were based on the inbred strains LEW and SHR, like the first study. Therefore, up to now, it was not possible to determine whether this QTL (and its corresponding gene or genes) would only be relevant to animals derived from these specific parental strains, or would also prove important for other populations of laboratory rats, standing out as a promising target to be investigated in other animal species, including humans.

Like in the original study (Ramos et al., 1999), the microsatellite markers D4RAT59 and D4MGH27, flanking
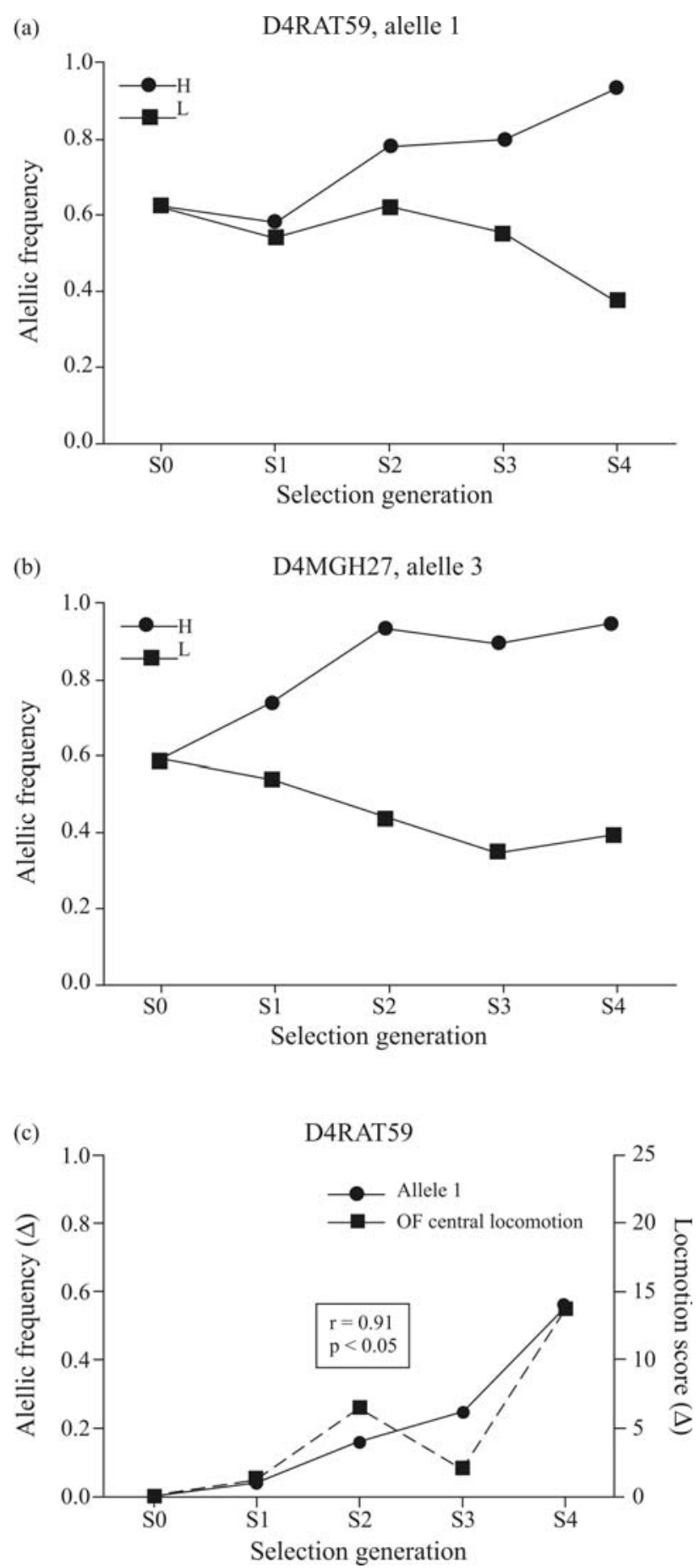

Figure 2 - Comparative frequencies ((a) and (b)) of allele 1 of D4RAT59 and allele 3 of D4MGH27 between the Floripa $\mathrm{H}$ and $\mathrm{L}$ rat lines, from generation S0 to S4 of selective breeding. Correlation (c) between the differences in behavioral scores for central locomotion in the OF test and the differences in the frequency of allele 1 of D4RAT59 between the Floripa $\mathrm{H}$ and $\mathrm{L}$ rat lines. Delta values for both variables were calculated, for each generation, as $\mathrm{H}-\mathrm{L}$. A significant correlation was found between the two variables. 
the peak of the QTL Ofill, were polymorphic in the present S0 population, presenting here three and two identifiable alleles, respectively. Based on the principle that the factors affecting the allelic frequencies in a population are mutation, migration, selection and random genetic drift, monitoring the allelic frequencies of the flanking markers of Ofill, from generation S0 to S4, would provide useful information on the possible association between Ofill and the behavioral trait of interest. The effects of mutation and migration could be discarded here, since rats of both lines, $\mathrm{H}$ and L, were only mated within lines and because the number of generations was far too small to allow significant direct effects of mutations (Falconer, 1987). Therefore, the influences of genetic drift and selection appeared here as the only possible factors to explain the changes in genotype and allele frequencies to be found across generations.

A similar experimental strategy was already successfully used in two different studies with mice (Buck et al., 1997; Kondaurova et al., 2006). Nevertheless, both these previous studies began their selective processes using two parental inbred strains which were known to be polymorphic for the QTLs and markers of interest, and were known to be different for the phenotype under study. Therefore, this strategy was much simpler and more easily interpretable than the one used in the present work, which was based on strains that were not inbred and had never been compared genotypically. On the other hand, as it used a more natural population, the results of the present strategy should provide a more general validation for the QTL and would be more meaningful as a model for the study of human disorders.

In the study by Ramos et al. (1999), the QTL Ofill was found to affect only females. In a second study by Mormède et al. (2002), however, the effects of this locus appeared in both sexes, and recent data from our laboratory (unpublished) confirmed that this locus can have an effect on males. In the present study, a preliminary analysis of the data, separately for each sex, indicated that the genotype results across generations were very similar for males and females. Therefore, as the present sample sizes were small, data from both sexes were pooled.

Like in the study by Kondaurova et al. (2006), we attempted to establish a link between Ofill and anxietyrelated behaviors using a chi-square test to verify the premises of the Hardy-Weinberg equilibrium. As expected, most of the generations $(10 / 16$, considering both markers and both $\mathrm{H}$ and $\mathrm{L}$ rat lines) were found to be significantly out of equilibrium, which is in agreement with the quite obvious changes in allelic frequencies illustrated in Figures 1 and 2 . In addition to that, we analyzed all generations simultaneously, using a contingency chi-square test. This analysis confirmed, indeed, that the successive generations of Floripa rats were very dissimilar when compared to each other, thus providing further statistical support to the genetic changes observed in both $\mathrm{H}$ and $\mathrm{L}$ rat lines during selection.
Figure 2 (panels a and b) shows very clearly that the allele 1 of marker D4RAT59 and the allele 3 of marker D4MGH27 presented a consistent increase in their frequencies during the selective breeding of $\mathrm{H}$ animals. As mentioned before, this fact alone could not be seen as a proof of selection, as it could also be explained by chance. Nevertheless, the fact that the Floripa L line, which evolved independently from the $\mathrm{H}$ line, mirrored the changes in the frequencies of the same two alleles provides support to the hypothesis that selection, rather than random genetic drift, was the major factor responsible for these changes. In addition, the original QTL study by Ramos et al. (1999) and the confirmatory study by Vendruscolo et al. (2006) both showed that alleles originated from the LEW rat strain caused an increase in central locomotion in the OF test in relation to the alleles inherited from the SHR strain at the locus Ofill, mapped between the markers D4RAT59 and D4MGH27. The direction of this effect is totally compatible with the changes in allele frequencies observed here, since in the present study a high frequency of alleles 1 (D4RAT59) and 3 (D4MGH27), both present in LEW rats, was associated with a high locomotion in the OF central area in the Floripa $\mathrm{H}$ rat line, as shown in Figure 2.

Finally, we analyzed the correlations between the present changes in allele frequencies and the changes in the selected phenotype previously reported by Ramos et al. (2003). Since the alleles 1 of D4RAT59 and 3 of D4MGH27 showed the most evident divergence during the selection process, we used the inter-line variation in their frequencies as compared to the inter-line variations in behavior. This H-L difference was adopted in this analysis in an attempt to minimize the uncontrolled effects of the environment on the behavior of the different generations, which varied not only genetically but also in time of the year, human observer and other minor differences in the laboratory environment. Such effects may have influenced the results of behavioral testing independently of the genotype, making behavior to fluctuate across generations in both $\mathrm{H}$ and $\mathrm{L}$ lines (Ramos et al., 2003). The present results show that the inter-line changes in the frequency of allele 1 for D4RAT59, from S0 to S4, were significantly correlated with the between-line differences in the central locomotion in the $\mathrm{OF}$ test, thus further corroborating the hypothesis that the genome region around Ofill contains one or more genes influencing this behavior. As the correlation was not significant for D4MGH27, our results might suggest that the gene(s) involved are closer to D4RAT59 than to D4MGH27. However, these results should be considered with caution because of the small number of data points (only five) used in the correlation analyses. Such a small sample size may provide false-negative results and thus the present experimental data may require further confirmation.

According to the linkage map developed by Ramos et al. (1999), D4RAT59 and D4MGH27 had a distance of 
$13.4 \mathrm{cM}$ between them, with the peak of the QTL being slightly closer to D4MGH27. The current molecular rat map (Rat Genome Database) indicates that there are near 20.8 million bases between the two markers. Thus, taking these positions as being much more precise than those provided by all previous linkage rat maps, the present results suggest that the gene(s) responsible for the effects of Ofill are less than $10 \mathrm{Mb}$ apart from D4RAT59. Additional genetic studies are under way in our laboratory to further refine the mapping of Ofil1.

Several other behavioral QTLs have been mapped to rat chromosome 4 in the vicinity of Ofill, such as Alc14, Alc16 and Alc18 for alcohol intake and preference, and Fit 1 for food intake (Rat Genome Database; Bice et al., 1998; Carr et al., 1998; Terenina-Rigaldie et al., 2003). Near this region, several candidate genes have been identified as potentially important for the central nervous system control of emotional behaviors, such as the genes for GABA (gamma-aminobutyric acid), Grid2 (glutamate receptor, ionotropic delta 2), Grin $2 b$ (glutamate receptor, ionotropic beta 2), tyrotrophin releasing hormone, and synapsin 2 . Further studies should investigate the potential relevance of these genes in relation to the behavioral effects of Ofill.

The results of the present study suggest that selection for central locomotion in the OF test, an anxiety-related behavior, acted on the genotype frequencies of two markers located on rat chromosome 4, flanking Ofill. One of these markers showed a significant correlation with the selected phenotype, which leads to the suggestion that this phenotype is influenced by the genotypic variation around Ofill. Thus, this region of the genome must contain one or more genes contributing to an anxiety-related behavior in different rat populations, including the strains LEW, SHR and Floripa $\mathrm{H}$ and $\mathrm{L}$. The further identification of the genes involved can be transposed to the human genome by homologous regions of the two species, enabling the study of the influence of these genes on anxiety-related disorders.

\section{Acknowledgments}

The authors wish to thank Dr Paulo R. P. Hofmann for helping with the data analysis, Dr Ilíada R. de Souza for providing technical and material support, and also Francine Borges, Gustavo R. Bruske and Elayne Pereira for giving technical assistance. Thais M. Hameister and Geison S. Izidio had scholarships from CAPES / Brazil, and André Ramos had a fellowship from CNPq / Brazil. This study was funded by the Universal grant 02/2003 from FAPESC and by the PRONEX grant 427/2003 from FAPESC-CNPq.

\section{References}

Anagnostaras SG, Craske MG and Fanselow MS (1999) Anxiety: At the intersection of genes and experience. Nat Neurosci 2:780-782.

Bice P, Foroud T, Bo R, Castelluccio P, Lumeng L, Li TK and Carr LG (1998) Genomic screen for QTLs underlying alco- hol consumption in the $\mathrm{P}$ and NP rat lines. Mamm Genome 9:949-955.

Buck KJ, Metten P, Belknap JK and Crabbe JC (1997) Quantitative trait loci involved in genetic predisposition to acute alcohol withdrawal in mice. J Neurosci 17:3946-3955.

Carr LG, Foroud T, Bice P, Gobbett T, Ivashina J, Edenberg H, Lumeng L and Li TK (1998) A quantitative trait locus for alcohol consumption in selectively bred rat lines. Alcohol Clin Exp Res 22:884-887.

Falconer DS (1987) Introdução à Genética Quantitativa. 1 edição. Imprensa Universitária da Universidade Federal de Viçosa, Viçosa, 279 pp.

Finn DA, Rutledge-Gorman MT and Crabbe JC (2003) Genetic animal models of anxiety. Neurogenetics 4:109-135.

Flint J (2003) Animal models of anxiety and their molecular dissection. Semin Cell Dev Biol 14:37-42.

Kondaurova EM, Bazovkina DV, Kulikov AV and Popova NK (2006) Selective breeding for catalepsy changes the distribution of microsatellite D13Mit76 alleles linked to the 5HT1A serotonin receptor gene in mice. Genes Brain Behav 5:596-601.

Mormède $\mathrm{P}$, Moneva E, Bruneval C, Chaouloff F and Moisan MP (2002) Marker-assisted selection of a neuro-behavioural trait related to behavioural inhibition in the SHR strain, an animal model of ADHD. Genes Brain Behav 1:111-116.

Potenza MN, Brodkin ES, Joe B, Lou X, Remmers EF, Wilder RL, Nestler EJ and Garlernter J (2004) Genomic regions controlling corticosterone levels in rats. Biol Psychiatry 55:634-641.

Ramos A and Mormède P (1998) Stress and emotionality: A multidimensional and genetic approach. Neurosci Biobehav Rev 22:33-57.

Ramos A, Moisan MP, Chaouloff F, Mormède $\mathrm{C}$ and Mormède $\mathrm{P}$ (1999) Identification of female-specific QTLs affecting an emotionality-related behavior in rats. Mol Psychiatry 4:453-462.

Ramos A, Correia EC, Izídio GS and Brüske GR (2003) Genetic selection of two new rat lines displaying different levels of anxiety-related behaviors. Behav Genet 33:657-668.

Terenina-Rigaldie E, Moisan MP, Colas A, Beaugé F, Shah KV, Jones BC and Mormède P (2003) Genetics of behaviour: Phenotypic and molecular study of rats derived from highand low-alcohol consuming lines. Pharmacogenetics 13:543-554.

Vendruscolo LF, Terenina-Rigaldie E, Raba F, Ramos A, Takahashi RN and Mormède P (2006) Evidence for femalespecific effect of chromosome 4 locus on anxiety-related behaviors and ethanol drinking in rats. Genes Brain Behav 5:441-450.

Willis-Owen SA and Flint J (2007) Identifying the genetic determinants of emotionality in humans; insights from rodents. Neurosci Biobehav Rev 31:115-124.

\section{Internet Resources:}

Rat Genome Database, http://rgd.mcw.edu/wg/ (January 31, 2008).

Associate Editor: Paulo A. Otto

License information: This is an open-access article distributed under the terms of the Creative Commons Attribution License, which permits unrestricted use, distribution, and reproduction in any medium, provided the original work is properly cited. 